\title{
Erratum to: Intraguild dietary overlap and its possible relationship to the coexistence of mesocarnivores in intensive agricultural habitats
}

\author{
Luigi Remonti • Alessandro Balestrieri • \\ Aritz Ruiz-González • Benjamín J. Gómez-Moliner • \\ Enrica Capelli · Claudio Prigioni
}

Published online: 30 August 2012

(C) The Society of Population Ecology and Springer 2012

\section{Erratum to: Popul Ecol}

\section{DOI 10.1007/s10144-012-0326-5}

In the original publication of the article, the word "Garbage" in Tables 1 and 2 has been erroneously published in bold.

Please find below the corrected version of Tables 1 and 2 .

The online version of the original article can be found under doi:10.1007/s10144-012-0326-5.

L. Remonti $(\bowtie) \cdot$ A. Balestrieri - E. Capelli · C. Prigioni Department of Earth and Environmental Sciences,

University of Pavia, Via Taramelli 24, 27100 Pavia, Italy e-mail: luigi.remonti@gmail.com
A. Balestrieri
e-mail: alebls@libero.it
E. Capelli
e-mail: capelli@unipv.it
C. Prigioni
e-mail: prigioni@unipv.it

\begin{abstract}
A. Ruiz-González · B. J. Gómez-Moliner
Department of Zoology and Animal Cell Biology,

University of the Basque Country, UPV/EHU;

C/Paseo de la Universidad 7, 01006 Vitoria-Gasteiz, Spain

e-mail: aritz.ruiz@ehu.es
\end{abstract}

B. J. Gómez-Moliner

e-mail: benjamin.gomez@ehu.es

\author{
A. Ruiz-González · B. J. Gómez-Moliner \\ Systematics, Biogeography and Population Dynamics Research \\ Group, CIEA "Lucio Lascaray", Centre for Research and \\ Advanced Studies, UPV/EHU; Avda Miguel de Unamuno, 3, \\ 01006 Vitoria-Gasteiz, Spain
}


Table 1 Results of the analysis of stone marten Martes foina and red fox 2 Vulpes vulpes faeces from the study sites of Camino and Coniolo, northern Italy

\begin{tabular}{|c|c|c|c|c|}
\hline \multirow[t]{2}{*}{ Food item } & \multicolumn{2}{|c|}{ Stone marten } & \multicolumn{2}{|c|}{ Red fox 2} \\
\hline & $\% F O$ & $\% m V$ & $\% F O$ & $\% m V$ \\
\hline Undetermined vegetal matter & 1.1 & 1.1 & 0 & 0 \\
\hline Fruits & 63.7 & 56.1 & 70.5 & 59.7 \\
\hline $\begin{array}{l}\text { American pokeweed Phytolacca } \\
\text { americana }\end{array}$ & 0 & 0 & 0 & 0 \\
\hline Black mulberry Morus nigra & 0 & 0 & 0 & 0 \\
\hline Berries Rubus sp. & 1.1 & 1.1 & 1.3 & 0.4 \\
\hline Wild cherry Prunus sp. & 37.4 & 32.1 & 60.3 & 50.4 \\
\hline Domestic cherry Prunus avium & 12.1 & 11.3 & 0 & 0 \\
\hline Plums Prunus domestica & 2.2 & 2.2 & 1.3 & 0.6 \\
\hline Pear Pyrus sp. & 0 & 0 & 0 & 0 \\
\hline Black elder Sambucus nigra & 0 & 0 & 0 & 0 \\
\hline Common grape vine Vitis vinifera & 0 & 0 & 0 & 0 \\
\hline Common fig Ficus carica & 6.6 & 5.7 & 6.4 & 5.3 \\
\hline Undetermined fruit & 1.1 & 0.5 & 3.8 & 1.9 \\
\hline Insects & 15.4 & 7.9 & 35.9 & 15.4 \\
\hline Lepidoptera larvae & 0 & 0 & 0 & 0 \\
\hline Coleoptera larvae & 2.2 & 2.2 & 0 & 0 \\
\hline Hymenoptera & 0 & 0 & 5.1 & 1.5 \\
\hline Orthoptera & 1.1 & 0.1 & 0 & 0 \\
\hline Coleoptera & 12.1 & 5.6 & 30.8 & 14.0 \\
\hline Undetermined insect & 0 & 0 & 0 & 0 \\
\hline Birds & 15.4 & 13.6 & 6.4 & 2.9 \\
\hline Anseriformes & 0 & 0 & 0 & 0 \\
\hline Galliformes & 0 & 0 & 0 & 0 \\
\hline Passeriformes & 14.3 & 12.9 & 6.4 & 2.9 \\
\hline Columbiformes & 1.1 & 0.8 & 0 & 0 \\
\hline Rallidae & 0 & 0 & 0 & 0 \\
\hline Undetermined bird & 0 & 0 & 0 & 0 \\
\hline Mammals & 24.2 & 20.4 & 24.4 & 18.8 \\
\hline Small mammals & 13.2 & 10.3 & 16.7 & 11.9 \\
\hline Common shrew Sorex araneus & 0 & 0 & 0 & 0 \\
\hline Fat dormouse Myoxus glis & 0 & 0 & 0 & 0 \\
\hline $\begin{array}{l}\text { Common dormouse } \\
\text { M. avellanarius }\end{array}$ & 0 & 0 & 1.3 & 0.8 \\
\hline Bank vole Myodes glareolus & 3.3 & 2.3 & 7.7 & 5.1 \\
\hline Savi's pine vole Microtus savii & 0 & 0 & 0 & 0 \\
\hline Water vole Arvicola terrestris & 0 & 0 & 0 & 0 \\
\hline Rat Rattus sp. & 3.3 & 3.3 & 5.1 & 5.1 \\
\hline Wood mouse Apodemus sp. & 3.3 & 2.5 & 0 & 0 \\
\hline House mouse Mus domesticus & 0 & 0 & 1.3 & 0.4 \\
\hline Undetermined rodent & 3.3 & 2.2 & 1.3 & 0.5 \\
\hline Medium-sized mammals & 9.9 & 9.0 & 9.0 & 6.9 \\
\hline $\begin{array}{l}\text { Eastern cottontail Sylvilagus } \\
\text { floridanus }\end{array}$ & 7.7 & 6.9 & 9.0 & 6.9 \\
\hline Brown hare Lepus europaeus & 1.1 & 1.1 & 0 & 0 \\
\hline Undetermined lagomorph & 1.1 & 1.0 & 0 & 0 \\
\hline
\end{tabular}

Table 1 continued

\begin{tabular}{|c|c|c|c|c|}
\hline \multirow[t]{2}{*}{ Food item } & \multicolumn{2}{|c|}{ Stone marten } & \multicolumn{2}{|c|}{ Red fox 2} \\
\hline & $\% F O$ & $\% m V$ & $\% F O$ & $\% m V$ \\
\hline Marten Martes sp. & 0 & 0 & 0 & 0 \\
\hline Large mammals & 1.1 & 1.1 & 0 & 0 \\
\hline Coypu Myocastor coypus & 1.1 & 1.1 & 0 & 0 \\
\hline Dog Canis domesticus & 0 & 0 & 0 & 0 \\
\hline Wild boar Sus scrofa & 0 & 0 & 0 & 0 \\
\hline Garbage & 0 & 0 & 2.6 & 2.6 \\
\hline
\end{tabular}

Data were available only for the warm season

In bold the food items excluded from the calculation of niche breadth and overlap 
Table 2 Results of the analysis of pine marten Martes martes and red fox 1 Vulpes vulpes faeces from the study sites of Valenza and San Massimo, northern Italy

\begin{tabular}{|c|c|c|c|c|c|c|c|c|}
\hline \multirow[t]{3}{*}{ Food item } & \multicolumn{4}{|c|}{ Pine marten } & \multicolumn{4}{|c|}{ Red fox 1} \\
\hline & \multicolumn{2}{|c|}{ Warm season } & \multicolumn{2}{|c|}{ Cold season } & \multicolumn{2}{|c|}{ Warm season } & \multicolumn{2}{|c|}{ Cold season } \\
\hline & $\% F O$ & $\% m V$ & $\% F O$ & $\% m V$ & $\% F O$ & $\% m V$ & $\% F O$ & $\% m V$ \\
\hline Undetermined vegetal matter & 4.8 & 3.5 & 4.4 & 3.7 & 1.8 & 1.8 & 0 & 0 \\
\hline Fruits & 58.7 & 47.1 & 8.7 & 4.0 & 35.1 & 26.1 & 10.5 & 3.9 \\
\hline American pokeweed Phytolacca americana & 1.6 & 0.3 & 0 & 0 & 0 & 0 & 7.0 & 1.8 \\
\hline Black mulberry Morus nigra & 9.5 & 6.7 & 0 & 0 & 0 & 0 & 0 & 0 \\
\hline Berries Rubus sp. & 19.0 & 15.7 & 0 & 0 & 14.0 & 8.2 & 1.8 & 1.8 \\
\hline Wild cherry Prunus sp. & 22.2 & 14.9 & 0 & 0 & 5.3 & 2.5 & 0 & 0 \\
\hline Domestic cherry Prunus avium & 0 & 0 & 0 & 0 & 0 & 0 & 0 & 0 \\
\hline Plums Prunus domestica & 0 & 0 & 0 & 0 & 1.8 & 1.8 & 0 & 0 \\
\hline Pear Pyrus sp. & 6.3 & 4.1 & 0 & 0 & 7.0 & 3.9 & 0 & 0 \\
\hline Black elder Sambucus nigra & 0 & 0 & 0 & 0 & 3.5 & 1.9 & 1.8 & 0.2 \\
\hline Common grape vine Vitis vinifera & 0 & 0 & 2.2 & 0.3 & 3.5 & 1.2 & 0 & 0 \\
\hline Common fig Ficus carica & 4.8 & 3.7 & 2.2 & 1.1 & 8.8 & 6.3 & 0 & 0 \\
\hline Undetermined fruit & 1.6 & 1.6 & 4.4 & 2.6 & 1.8 & 0.4 & 1.8 & 0.2 \\
\hline Insects & 6.3 & 4.0 & 4.6 & 0.9 & 26.3 & 11.1 & 15.8 & 7.9 \\
\hline Lepidoptera larvae & 0 & 0 & 0 & 0 & 0 & 0 & 0 & 0 \\
\hline Coleoptera larvae & 3.2 & 1.7 & 0 & 0 & 1.8 & 1.1 & 10.5 & 5.8 \\
\hline Hymenoptera & 0 & 0 & 2.2 & 0.7 & 3.5 & 0.4 & 0 & 0 \\
\hline Orthoptera & 0 & 0 & 2.2 & 0.2 & 5.3 & 1.2 & 0 & 0 \\
\hline Coleoptera & 3.2 & 2.4 & 0 & 0 & 21.1 & 8.2 & 7.0 & 2.1 \\
\hline Undetermined insect & 0 & 0 & 0 & 0 & 1.8 & 0.4 & 0 & 0 \\
\hline Birds & 17.5 & 10.7 & 30.4 & 22.1 & 24.6 & 15.4 & 29.8 & 17.4 \\
\hline Anseriformes & 0 & 0 & 0 & 0 & 3.5 & 1.4 & 0 & 0 \\
\hline Galliformes & 0 & 0 & 4.4 & 3.0 & 5.3 & 4.0 & 3.5 & 3.5 \\
\hline Passeriformes & 15.9 & 9.6 & 13.0 & 7.2 & 8.8 & 5.3 & 15.8 & 8.2 \\
\hline Columbiformes & 1.6 & 1.1 & 10.9 & 9.8 & 5.3 & 3.9 & 7.0 & 3.7 \\
\hline Rallidae & 0 & 0 & 0 & 0 & 0 & 0 & 3.5 & 1.9 \\
\hline Undetermined bird & 0 & 0 & 2.2 & 2.2 & 1.8 & 0.7 & 0 & 0 \\
\hline Mammals & 41.3 & 34.7 & 76.1 & 67.2 & 52.6 & 43.6 & 78.9 & 65.3 \\
\hline Small mammals & 17.5 & 13.5 & 47.8 & 42.4 & 21.1 & 13.4 & 52.6 & 41.1 \\
\hline Common shrew Sorex araneus & 0 & 0 & 2.2 & 0.9 & 1.8 & 0.4 & 0 & 0 \\
\hline Fat dormouse Myoxus glis & 4.8 & 3.0 & 4.3 & 2.8 & 1.8 & 1.8 & 0 & 0 \\
\hline Common dormouse $M$. avellanarius & 1.6 & 1.6 & 0 & 0 & 0 & 0 & 0 & 0 \\
\hline Bank vole Myodes glareolus & 6.3 & 3.2 & 32.6 & 30.9 & 7.0 & 4.6 & 5.3 & 4.0 \\
\hline Savi's pine vole Microtus savii & 6.3 & 4.0 & 2.2 & 2.2 & 0 & 0 & 0 & 0 \\
\hline Water vole Arvicola terrestris & 4.8 & 4.8 & 0 & 0 & 5.3 & 4.7 & 10.5 & 8.4 \\
\hline Rat Rattus sp. & 0 & 0 & 0 & 0 & 0 & 0 & 8.8 & 8.2 \\
\hline Wood mouse Apodemus sp. & 0 & 0 & 2.2 & 2.2 & 3.5 & 1.4 & 17.5 & 12.1 \\
\hline House mouse Mus domesticus & 0 & 0 & 8.7 & 6.5 & 0 & 0 & 0 & 0 \\
\hline Undetermined rodent & 0 & 0 & 0 & 0 & 1.8 & 0.5 & 10.5 & 8.2 \\
\hline Medium-sized mammals & 20.6 & 18.2 & 19.6 & 17.0 & 26.3 & 22.3 & 24.6 & 20 \\
\hline Eastern cottontail Sylvilagus floridanus & 9.5 & 9.3 & 10.9 & 8.7 & 10.5 & 9.6 & 1.8 & 1.8 \\
\hline Brown hare Lepus europaeus & 7.9 & 6.2 & 8.7 & 8.3 & 12.3 & 9.1 & 26.3 & 20.2 \\
\hline Undetermined lagomorph & 3.2 & 2.7 & 0 & 0 & 0 & 0 & 0 & 0 \\
\hline Marten Martes sp. & 0 & 0 & 0 & 0 & 3.5 & 3.5 & 0 & 0 \\
\hline
\end{tabular}


Table 2 continued

\begin{tabular}{|c|c|c|c|c|c|c|c|c|}
\hline \multirow[t]{3}{*}{ Food item } & \multicolumn{4}{|c|}{ Pine marten } & \multicolumn{4}{|c|}{ Red fox 1} \\
\hline & \multicolumn{2}{|c|}{ Warm season } & \multicolumn{2}{|c|}{ Cold season } & \multicolumn{2}{|c|}{ Warm season } & \multicolumn{2}{|c|}{ Cold season } \\
\hline & $\% F O$ & $\% m V$ & $\% F O$ & $\% m V$ & $\% F O$ & $\% m V$ & $\% F O$ & $\% m V$ \\
\hline Large mammals & 0 & 0 & 6.5 & 5.4 & 8.8 & 8.2 & 8.8 & 7.9 \\
\hline Coypu Myocastor coypus & 0 & 0 & 8.7 & 7.0 & 7.0 & 6.5 & 7.0 & 6.1 \\
\hline Dog Canis domesticus & 0 & 0 & 0 & 0 & 0 & 0 & 0 & 0 \\
\hline Wild boar Sus scrofa & 0 & 0 & 0 & 0 & 1.8 & 1.8 & 1.8 & 1.8 \\
\hline Garbage & 0 & 0 & 0 & 0 & 1.8 & 1.8 & 0 & 0 \\
\hline
\end{tabular}

In bold, the food items excluded from the calculation of niche breadth and overlap 